\title{
Report of the \\ Panel on Leprosy Reaction
}

Chairman: F. Latapí (Mexico); Secretary:-1). S. Ridley (UK); Members: J. Ramos e Silva (Brazil); A. R. Mercau (Argentine); J. H. Frenken (Dutch Antilles); D. Pcryassú (Brazil); A. Aguirre de González (Paraguay); C. Sisiruca (Venezuela); C. K. Job (India); E. A. Carboni (Argentine); J. C. 'Tolentino (Phillippines); J. 'I'. De las Aguas (Spain).

The subject is very important. 'There is confusion in terminology.

\section{De finition}

In a broad sense the word reaction comprises all acute and subacute manifestations of leprosy. Leprologists are aware of the damages left by reactions in other forms of leprosy, but consider as more frequent and important acute and subacute episodes in lepromatous leprosy.

\section{Cilassification}

On this point the Madrid Congress classified as follows:

I. Lepra reaction

2. Erythema nodosum.

'This panel considers that the true lepra reaction is erythema nodosum and that the so-called 'lepra reaction' of the Madrid Congress should be called 'lepromatous exacerbation'.

It is recognised that there are objections to these terms. But it is suggested that in view of their long usage they should be retained until an improvement in the state of our knowledge makes it possible to adopt a rational nomenclaturc.

It is proposed therefore:

\section{Lepra reaction (lepromatous)}

An acute or subacute clinicopathological syndrome which appears during the chronic course of lepromatous leprosy with systemic symptoms and local lesions in the skin and other organs. They are related to varying degrees of vasculitis of an inflammatory non-granulomatous nature, which are produced by some mechanism of hypersensitivity whose pathogenesis needs to be clarified.

Lepra reaction presents a variety of clinical and histological manifestations which are broadly distinct but which frequently occur in combination - (e.g., erythema nodosum necrotisans).

(a) Erythema nodosum: Disseminated and painful nodosities, which appear in sites apparently not affected previously by the lepromatous process. 
(b) Erythema multiforme: The typical appearance is of flat and extensive reddish lesions.

(c) Erythema necrotisans: (I,ucio phenomenon). Multiple red and painful capriciously shaped spots with a tendency to dry or bullous necrosis. This cutancous lesion of lepra reaction is more common in diffuse lepromatous leprosy but may appear in the nodular form.

\section{Lepromatous exacerbation}

This term applies to a rapid extension of lepromatous lesions, with possible appearance of new ones of the same nature.

H I S T O L O G Y

\section{Lepra reaction:}

(a) Erythema nodosum

(b) Erythema multiforme.

The characteristic of these reactions, which are histologically similar, is the focalised accumulation of polymorphs in the dermis or subcutaneous tissue. The lepromatous granuloma, usually small, is regressive with much foamy degeneration. The dermis is otherwise normal.

(c) Erythema nodosum necrotisans.

The extensive polymorph infiltration sometimes proceeds to abscess formation. It is more diffuse than in other types of lepra reaction. Capillaries frequently show endothelial swelling and sometimes necrosis. Towards the end of the reaction there is an increase of plasma cells.

Oedema is a prominent feature of this reaction. The dermis shows evidence of collagen and elastic damage, with fibroblastic increase.

(d) Lucio phenomenon.

Polymorph infiltration is intense. It is diffuse or multi-focal. The reaction is characterised by vasculitis, the vessels affected being of larger calibre than in necrotising ENI. The dermis is not much affected.

\section{Lepromatous exacerbation}

The reaction is mild. There is some oedema. The dermis shows a slight increase of fibroblasts. The lepromatous granuloma differs from that of lepra reaction: it is active, with an increase of histiocytes and early macrophages.

\section{Lepra reaction}

BA CTERIOL OGY

Bacilli are usually few in the areas of polymorph infiltration. In the surrounding lepromatous foci the number of bacilli is comparable to that in the non-reacting lepromatous lesions.

The bacilli are already granular before the onset of the reaction.

\section{Lepromatous exacerbation}

There is an increase in the number of bacilli in the lesions at the time of the reaction, and an increase in the percentage of solid-staining forms. 
The acute and subacute episodes are closely linked with the immunological process which in turn determines the various clinical forms of leprosy. 'The disturbance of the immunological equilibrium may precipitate an acute attack with the appearance of disseminated lesions. The discase itself may become better or worse.

There are several factors that may disturb the equilibrium between the host and the bacilli. Physical and mental stress, endocrine imbalance, intercurrent infections, specific antileprosy therapy, are some of the more important ones.

Some others think that these acute and subacute episodes are part of the natural history of the discase independent of external factors.

It is suggested that intensive research in immunology be undertaken to clucidate the pathogenesis of this complex process.

\section{TREATMEN T}

Bearing in mind the various factors liable to intervene in the pathogenesis of leprosy reaction, rational therapy should be mainly designed to eliminate or interfere with the constant or repeated action of the determining or 'trigger' causes referred to above.

Likewise, treatment shall be conditioned in each case to the severity of the reactional episodes.

To this end, therapy will be directed as follows:

(a) Basic general treatment, intended to central systemic symptoms.

In such cases, emphasis is laid on the restoration of the blood picture, which is nearly always disturbed by a severe lepromatous reaction, by means of blood transfusion.

(b) Symptomatic treatment, specially designed to act on the acute, focal or regional manifestations, such as ocular, neural, articular, testicular and visceral reactions.

In such cases it is advisable to call in the corresponding specialists for consultation.

In the circumstances indicated above, as in others not specified, benefits can be secured by the use of the antimonials, certain antibiotics, the antimalarials, antihistaminics, etc.

Only when acute attacks are severe can the administration of corticoids or ACTH, under strict medical control, be considered. We advise against their routine use.

Specific treatment of leprosy shall be maintained, lessened or stopped altogether according to the severity of the reactional state.

\section{P R O P H Y L A X IS}

In this connection, we would make the following recommendations:

( I) Every patient, before specific treatment is started, shall be submitted to a comprehensive clinical examination.

The presence of any intercurrent affection of a general (infectious, metabolic, hormonal) or local (septic, foci, parasites, etc.) nature shall be discarded. 
A record shall be made of nutritional state, and also of any stress that might disturb his psychosomatic balance.

(2) $\Lambda$ detailed laboratory examination, specially of blood and serum, shall likewise be made.

(3) Specific treatment shall not be started until the patient is found to be in good condition.

(4) Initial doses shall be minimal, reaching the effective dosage only after a suitable period.

(5) Periodical clinical and laboratory examination will be made as frequently as required in each particular case.

(6) 'The unsuitability of iodide medication should be borne in mind. 